\title{
Concordance: A Critical Participatory Alternative in Healthcare IT
}

\author{
Erik Grönvall \\ IT Uni. of Copenhagen \\ Rued Langgaards Vej 7 \\ Copenhagen, Denmark \\ erig@itu.dk
}

\author{
Nervo Verdezoto \\ Aarhus University \\ Aabogade 34 D, 8200 \\ Aarhus, Denmark \\ nervo@cs.au.dk
}

\author{
Naveen Bagalkot \\ Srishti School of Art, \\ Design and Tech., \\ Bangalore, India \\ naveen@srishti.ac.in
}

\author{
Tomas Sokoler \\ IT Uni. of Copenhagen \\ Rued Langgaards Vej 7 \\ Copenhagen, Denmark \\ sokoler@itu.dk
}

\begin{abstract}
The healthcare sector is undergoing large changes in which technology is given a more active role in both in-clinic and out-of-clinic care. Authoritative healthcare models such as compliance and adherence which relies on asymmetric patient-doctor relationships are being challenged as society, patient roles and care contexts transforms, for example when care activities move into non-clinical contexts. Concordance is an alternative model proposed by the medical field that favours an equal and collaborative patient-doctor relationship in the negotiation of care. Similarly, HCI researchers have applied diverse models of engagement in IT design ranging from authoritative models (e.g. perceiving people as human factors to design for) to more democratic design processes (e.g. Participatory Design). IT design has also been crafted as on-going processes that are integrated parts of everyday use. Based on the best practice of participation from the medical and the HCI fields, we identify critical alternatives for healthcare design. These alternatives highlight opportunities with ongoing design processes in which the design of care regimens and care IT are perceived as one process.
\end{abstract}

\section{Author Keywords \\ Concordance; Critical Alternative; Participatory Design; Healthcare; Ongoing design; Infrastructuring}

\section{ACM Classification Keywords}

H.5.m. Information interfaces and presentation (e.g., HCI): Miscellaneous.

\section{INTRODUCTION}

During the last decade the doctor-patient relationship has been redefined. It has undergone a transformation from being defined by authoritative care models towards models with a more balanced relationship between care providers and receivers. Information Technology (IT) systems for healthcare and their design processes may either reinforce the authoritative care models or facilitate a more equal

Copyright $\subset 2015$ is held by the author(s). Publication rights licensed to Aarhus University and ACM

5th Decennial Aarhus Conference on Critical Alternatives August 17 -21, 2015, Aarhus Denmark collaboration between patients and healthcare professionals in the negotiation of care activities. The HCI field has also gone through a transformation where the perception and role of the 'user' and other stakeholders in design has changed. People have gone from being perceived as human factors, to users, to design collaborators, and lately as active participants in design processes that design-for-future-use (rather than design-for-use) [3-5]. Ongoing design processes include a shift away from design-for-use towards designing for design after design. Here, infrastructuring is used within the design literature (see e.g. Ehn [5] and Seravelli [16]), to explore how to design meta-designs, or designing for design after design, and how diverse stakeholders through constantly ongoing design processes design-in-use rather than designing for use before use.

Reflecting on the above transformations, and our own experiences working with healthcare IT and design, we describe perspectives on participation originated from both the medical and the HCI field. From the medical field, we take the transformation from compliance and adherence to concordance to enlighten the perspectives on participation. In contrast to compliance and adherence that represent authoritative models of care where the patient is perceived as a passive receiver of care instructions [13], concordancebased care emphasizes an active patient, co-responsible in defining and ensuring their own care plans. From the HCI field, we account for 'ongoing design processes' and 'participatory design' as design ideals. Based on these ideals from the medical (i.e. concordance) and design (i.e. PD and infrastructuring) fields, and in particular their roles in contemporary healthcare IT projects, we show how bridging disciplinary boundaries could offer critical alternatives on how to design for future care and care IT.

\section{PARTICIPATION FROM A HEALTHCARE AND DESIGN PERSPECTIVE}

Considering the shifting notions of participation within HCI and healthcare, it seems important to examine the roles that various actors may assume in healthcare IT design and use and how participation is shaping current and future care practices. We will now describe participation and how it has been perceived and changed in healthcare as well as in healthcare IT design processes. 
Participation in Healthcare: From Compliance to Concordance

Compliance has traditionally been the prevailing relationship between a doctor and her patients. Compliance measures to what degree a patient follows a prescribed treatment, without considering the patient as a partner in the delivery of care [13]. However, as reported in the medical literature (e.g. [13]), a prescription that a patient disagrees with already at the medical consultation will have less or no value. Therefore a shift from compliance to adherence was initiated within the medical practice. Adherence extends compliance by also considering to what degree a patient agrees with the proposed treatment plan. However, both compliance and adherence have been criticized within the medical field as being too authoritative [9]. The medical field has proposed concordance as a more democratic model to shape the doctor-patient relationship. Concordance emphasizes equal participation and active doctor-patient collaboration at the clinic in both the definition and fulfilment of a care regimen [9]. In doing so, concordance promotes an ongoing negotiation between the patient and healthcare professional through a democratic and open-ended patient-professional collaboration process [9]. As such, concordance involves a political stance where the patient and healthcare professional are considered equal peers in the treatment.

From a medical perspective, concordance is an activity situated at the clinic, as part of the medical consultation. However, in an earlier work by the authors, it is argued that the implementation of care activities in everyday life situations extends beyond the clinic. The negotiation of care is situated in everyday life contexts and should be considered as an ongoing negotiation [2]. Our previous work showed how care receivers negotiate the integration of prescribed assistive tools into their home environment and the impact of the care regimen on them and their families' everyday lives. For example, a woman rejected a prescribed stepping board, as she preferred to use her own stairs for her physiotherapy. Another example was a spouse that embedded objects from the home environment into the patient's rehabilitation activities to support the prescribed exercises. These examples illustrate the patients' ongoing negotiations with others, objects and the environments to manage their out-of-clinic care. The examples also extend the role of concordance to include more than the in-clinic consultation activities, embracing also the ongoing healthcare practices that take place in everyday life.

Meanwhile, concordance is by no means a settled debate in the medical discourses. Its focus on changing the responsibilities of the doctors and patients is critical, and it is argued that the consequences of increased patient responsibility for their own care needs further exploration from a societal, moral and ethical perspective. In particular, Segal states that healthcare professionals should intervene if patients with life-threatening or very contagious diseases refuse antibiotics or other treatments [15]. As concordance is moved out of the clinical consultations and into the people's everyday settings and routines, the discussion about patients' and doctors' roles and responsibilities become even more critical.

\section{Participation in Design: Participatory Design in Healthcare}

Within HCI, there has been a shift during the last 30 years from perceiving people as human factors to consider them as actors within the design process $[3,7,10]$. Today many HCI practitioners acknowledge the benefits of involving different stakeholders in design processes. Participatory Design (PD) is one design approach based on an active and equal involvement of different stakeholders throughout the design process. Initially, much of the PD work in healthcare focused on initiatives coming from professional settings to support the development process of technology focusing on both patient's and health professional's needs to improve clinical practice. For example, developing Electronic Patient Record systems to support clinical consultations and medical practices [18]. In recent years, the increasing move of care from the hospital to the home has required the application of diverse PD methods in non-professional settings to actively involve patients in the design process, uncovering their specific needs in relation to their treatments and their everyday life [7, 12, 24]. In nonprofessional settings, uncovered challenges for patients to perform care activities includes the lack of patient's motivation to perform the prescribed treatment, the individual mental and physical capabilities, the individual's perception of technology, the dynamic use context, the aesthetics of the home and of care technology, and the distribution of control over the setting [7].

An ever-growing number of healthcare IT design and research projects have applied participatory methods to engage patients and doctors in designing healthcare IT systems for later deployment and use. Contemporary PD research do not only consider how to design ready-to-use systems but also investigates meta-designs and designing for design after design (i.e. infrastructuring) to allow people to participate in ongoing design processes (design-in-use) [5]. As such, infrastructuring calls for attention to the active role of people as designers, through use, to develop support for practices that could not be envisioned before use [16].

While participatory healthcare IT researchers has eagerly adopted the ideals of active participation of patients and doctors in the design process (e.g., [7, 22]), they have in general not considered the role of patients' active participation in shaping their own care regimen after the initial design process has ended, nor designed for such participation. In other words, many healthcare IT designs have adopted the medical ideals of compliance and adherence, designing healthcare IT solutions that ensure or support a patient to follow a prescribed regimen (such as tools for remote-monitoring $[8,12,17]$ and medical reminders $[14,23])$. Herein lays a paradox. It seems that 
healthcare IT projects, while driven by methodological approaches that in their very essence build on models that value an active participation of different stakeholders (i.e., patients), are stuck with authoritative de-contextualized models concerning the relationship between doctor, patient, and treatment. Participatory design driven healthcare IT projects do exist that have investigated alternative strategies for care, including a renegotiated patient-physician relation and collaboration [1]. Nevertheless, with only few recent exceptions (e.g. [2]) concordance has not been a design goal in healthcare HCI projects [6]. Indeed, the development in the medical field towards concordance as an alternative to compliance and adherence has largely been overlooked by the healthcare IT design community. Instead, the HCI community has contributed for example with ideas of gamification and persuasive design to support the existing compliance and adherence models [14].

To critically rethink citizen participation in care and healthcare IT design, and the relationship between designing individualized care regimens and designing healthcare IT to support these regimens, may open up for new strategies to improve the quality of care. Also it may inform both the healthcare and design research fields.

\section{CRITICAL PARTICIPATORY ALTERNATIVES FOR HEALTHCARE IT DESIGN}

As presented above, many PD healthcare IT projects have been informed by a medical perspective but have not considered the unexpected ways of how people appropriate technology in relation to everyday practices [2]. However, there are critical alternatives to consider when designing future healthcare IT solutions within both contemporary Participatory Design and medical research fields. We are referring in particular to, firstly how PD research has investigated ongoing design processes, supported by infrastructuring [5], and secondly how the medical science has turned towards concordance as an alternative to patient compliance and adherence [9]. People negotiate and appropriate care activities and healthcare IT to make them part of their everyday lives [2, 20]. Rather than designing for a specific use, it may therefore be advantageous to support practices of appropriation and negotiation.

There are at least two possible developments in which PD research and the notion of concordance may join forces to provide a critical alternative for future healthcare designs. The first strand (A) is based upon two sequential phases. In phase one PD is used to co-design healthcare IT that aim for concordance rather than compliance and adherence (as we have previously argued in [2]). In the second phase (and as an extension to [2]) the design outcome of phase one is used by patients to negotiate and integrate concordance-based care practices into their everyday lives. The second strand (B) is an infrastructuring approach where the design of healthcare IT and the design of a patient's care regimen are conceptually considered equal and where both the design of IT and care is ongoing and perceived as one. These two development-strands represent critical alternatives to the dominant $\mathrm{PD}$ practice in healthcare where different stakeholders' co-design technology for later use under the dominance of compliance and adherence.

\section{Critical Alternatives: PD, Infrastructuring and Concordance}

Both PD and concordance involve a political stance where diverse stakeholders collaborate on democratic and equal terms. In particular, both approaches empower the citizen, being a (future) user of technology or receiver of care, to make an impact on his or her situation through active participation. Indeed, the philosophy behind PD and concordance bear much resemblance and, if thought of, there may not be such a large conceptual difference between co-designing one's own care (i.e. concordance) and co-designing the technology that will support the very same care. Or stated differently, should the design of healthcare IT and the design of one's care regimen be kept as two separate activities or should they be perceived as one? As the introduction of new technology into a given practice will also change the practice [11], an ongoing design of both IT and care seems favorable. Considering the two strands presented above, strand A may be more straightforward to implement and control (as compared to strand B) as there are two separate phases. Phase one focusing on healthcare technology design, and then in phase two individualized care activities are designed based on the technology from phase one. These two phases are tightly connected but clearly separated. In strand B, both the technology and care design are perceived as one ongoing design process based on the idea of infrastructuring. In strand B new possibilities exists (as compared to strand A) to challenge and inform both the PD and the medical fields. Applying an ongoing design approach (design-in-use) may be a way to discuss participation, facilitating care practices where both care and the care IT are co-designed over time. In sum, concordance and PD in concert can provide an alternative approach for designing healthcare IT, informing and strengthening each other.

\section{CONCLUSION}

In this paper, we have presented critical alternatives for future healthcare designs. The proposed alternatives move away from the authoritarian ideals of compliance and adherence towards a more participatory and collaborative doctor-patient relationship. This move implies that the separation of the design and the use of healthcare IT will dissolve. Through ongoing design processes, supported by concordance and infrastructuring, the act of designing individualized care regimens and designing healthcare IT to support these regimens may become one. The HCI research community should better understand the effects on the involved stakeholders (e.g. patients), not only working with concordance as a design ideal, but also working with ongoing design processes where both care regimens and healthcare technology are designed in concert. However, 
participation as discussed in this paper will require rather resourceful participants, something that should be taken into consideration in future work. These critical alternatives do not only have the potential to point to new directions for healthcare IT design targeting an individual patient. For example in Europe there is an increased interest on service co-creation within healthcare and patient involvement in designing healthcare services, as seen in national health policies and NGO initiatives $[19,21]$. It could prove useful to investigate ongoing design processes as described in this paper as a strategy for future healthcare services. The proposed critical alternatives can inspire designers and researchers to reconsider their design processes when designing out-of-clinic healthcare and technology to better support concordance-based care practices in people's everyday life.

\section{ACKNOWLEDGMENTS}

We like to thank colleagues from both the medical and HCI fields in formulating the thoughts expressed in this paper.

\section{REFERENCES}

1. Andersen, T., Bjørn, P., Kensing, F., et al. Designing for collaborative interpretation in telemonitoring: Reintroducing patients as diagnostic agents. International Journal of Medical Informatics, 2011, 80(8):e112-e126.

2. Bagalkot, N. L., Grönvall, E. and Sokoler, T., Concordance: design ideal for facilitating situated negotiations in out-of-clinic healthcare, In Proc. EA CHI '14, ACM Press(2014), 855-864.

3. Bannon, L., From human factors to human actors: the role of psychology and human-computer interaction studies in system design, In Design at work: cooperative design of computer systems, Lawrence Erlbaum Associates, Inc., Hillsdale, New Jersey, USA, 1991.

4. Dantec, C. L. and DiSalvo, C. Infrastructuring and the Formation of Publics in Participatory Design. Social Studies of Science, 2013, 43(2): 241-264.

5. Ehn, P., Participation in design things, In Proc. PDC 2008, ACM Press(2008), 92-101.

6. Fitzpatrick, G. and Ellingsen, G. A Review of 25 Years of CSCW Research in Healthcare: Contributions, Challenges and Future Agendas. Computer Supported Cooperative Work (CSCW), 2012, 22(4-6):609-665.

7. Grönvall, E. and Kyng, M. On participatory design of home-based healthcare. Cognition, Technology \& Work, 2013, 15(4):389-401.

8. Grönvall, E. and Verdezoto, N., Beyond SelfMonitoring: Understanding Non-functional Aspects of Home-based Healthcare Technology, In Proc. UbiComp 2013, ACM Press(2013), 587-596.

9. Horne, R., Weinman, J., Barber, N., et al. Concordance, adherence and compliance in medicine taking. London: NCCSDO, 2005.
10. Iversen, O. S., Kanstrup, A. M. and Petersen, M. G., A visit to the 'new Utopia': revitalizing democracy, emancipation and quality in co-operative design, In Proc. NordiCHI 2004, ACM Press(2004), 171-179.

11. Kaptelinin, V. and Bannon, L. J. Interaction Design Beyond the Product: Creating Technology-Enhanced Activity Spaces. Human-Computer Interaction, 2011, 27(3):277-309.

12. Kusk, K., Nielsen, D. B., Thylstrup, T., et al., Feasibility of using a lightweight context-aware system for facilitating reliable home blood pressure selfmeasurements, In Proc. PervasiveHealth 2013, IEEE (2013), 236-239.

13. Moore, K. N. Compliance or Collaboration? the Meaning for the Patient. Nursing Ethics, 1995, 2(1):7177.

14. Oliveira, R. d., Cherubini, M. and Oliver, N., MoviPill: improving medication compliance for elders using a mobile persuasive social game, In Proc. UBICOMP 2010, ACM Press(2010), 251-260.

15. Segal, J. "Compliance" to "Concordance": A Critical View. Journal of Medical Humanities, 2007, 28(2):8196.

16. Seravalli, A., Infrastructuring for opening production, from participatory design to participatory making?, In Proc. PDC 2012, ACM Press(2012), 53-56.

17. Siek, K., Khan, D., Ross, S., et al. Designing a Personal Health Application for Older Adults to Manage Medications: A Comprehensive Case Study. Journal of Medical Systems, 2011, 35(5):1099-1121.

18. Simonsen, J. and Hertzum, M., A Regional PD Strategy for EPR Systems: Evidence-Based IT Development, In Proc. PDC 2006-Vol II, 2006, 125-228.

19. Spencer, M., Dineen, R. and Phillips, A., Co-producing services - Co-creating health, NHS WALES, 2013.

20. Storni, C. Multiple Forms of Appropriation in SelfMonitoring Technology: Reflections on the Role of Evaluation in Future Self-Care. International Journal of Human-Computer Interaction, 2010, 26(5):537-561.

21. The health fundation, Co-creating Health, from: http://www.health.org.uk/programmes/co-creatinghealth, 2008.

22. Uzor, S., Baillie, L. and Skelton, D., Senior designers: empowering seniors to design enjoyable falls rehabilitation tools, In Proc. CHI 2012, ACM Press(2012), 1179-1188.

23. Vitality - GlowCaps, from: www.vitality.net, 2012.

24. Aarhus, R., Ballegaard, S. and Hansen, T., The eDiary: Bridging home and hospital through healthcare technology, In Proc ECSCW 2009, Springer(2009), 6383. 